\title{
Data Access on Tourism Resources Management System Based on Spring JDBC
}

\author{
Jifu Tong \\ Higher Professional Technical Institute, Bohai University, Jinzhou, 121013, China \\ tjf6161@126.com
}

Keywords: Spring JDBC; tourism resources management system; data access

\begin{abstract}
Tourism resource management system is a computer system for tourists, tour operators, travel managers and travel planners. The development of tourism has an important role. This paper based on the Spring JDBC to study data access technology for the system development. First, the Spring framework architecture is represented by the graph; secondly, JDBC access to the database process through a flow chart to show; and then, based on the DB2 database management system, carry out database logical structure design; and finally, Spring JDBC data access implementation, including the configuration of JDBC transaction manager, Spring inject the DataSource for DAO, entity classes construct and write DAO realization, and so on. The research content of this paper is to solve the key technical problems of the systems development, to improve the efficiency of systems development has great significance.
\end{abstract}

\section{Introduction}

Economic and social development, the progress of science and technology, real income growth, personal disposable leisure time increases, people innovation and learning, to seek happiness, health seeking desire enhancement, is the driving force behind the development of modern tourism. In order to meet the rapid development of China's tourism industry, a lot of tourism enterprises to establish a tourism information system, but there are still many problems in the construction, mainly not high in the overall research and application level, and information expression lacking vivid intuitive, object-oriented users single function, expression and analysis of spatial information is relatively weak, poor information exchange [1].

JDBC (Java Data Base Connectivity) is a Java API for executing SQL statements, which can be used to provide data access methods for a variety of relational database. Spring is an open source framework, the core technology is a lightweight container, providing full supporting application framework for J2EE-based enterprise-class application development [2]. When developing Java application based on Spring framework, there are two methods of data access, one is Spring JDBC, the other is the Spring Hibernate. This paper is based on Spring JDBC research data access, to provide support for the development of tourism resources management system.

\section{Architecture on Spring Framework}

Spring is a J2EE application framework, which is designed to solve the complexity of enterprise application development. It is a lightweight container framework of IoC and AOP, which can be used in the management of JavaBean's life cycle, can be used alone, can also be used in combination with Struts framework and iBatis framework. One of the main advantages of the Spring framework is the layered architecture, a layered architecture allows the user to select which components to use, as well as J2EE application provides integrated framework for the development. The use of Spring is not limited to server-side development. From the perspective of simplicity, testability, and loose coupling, any Java application can benefit from Spring. The core of the Spring is to control the inversion and aspect oriented. Spring is a layered JavaSE/EEfull-stack lightweight open-source framework. The core functionality of the Spring framework can be used in any Java application and it can also be used to build Web applications on the extended Java EE platform. The goal of the Spring framework to make J2EE development easier to use, and to promote good 
programming habits by making use of the POJO-based programming model. Spring framework architecture is shown in Fig. 1.

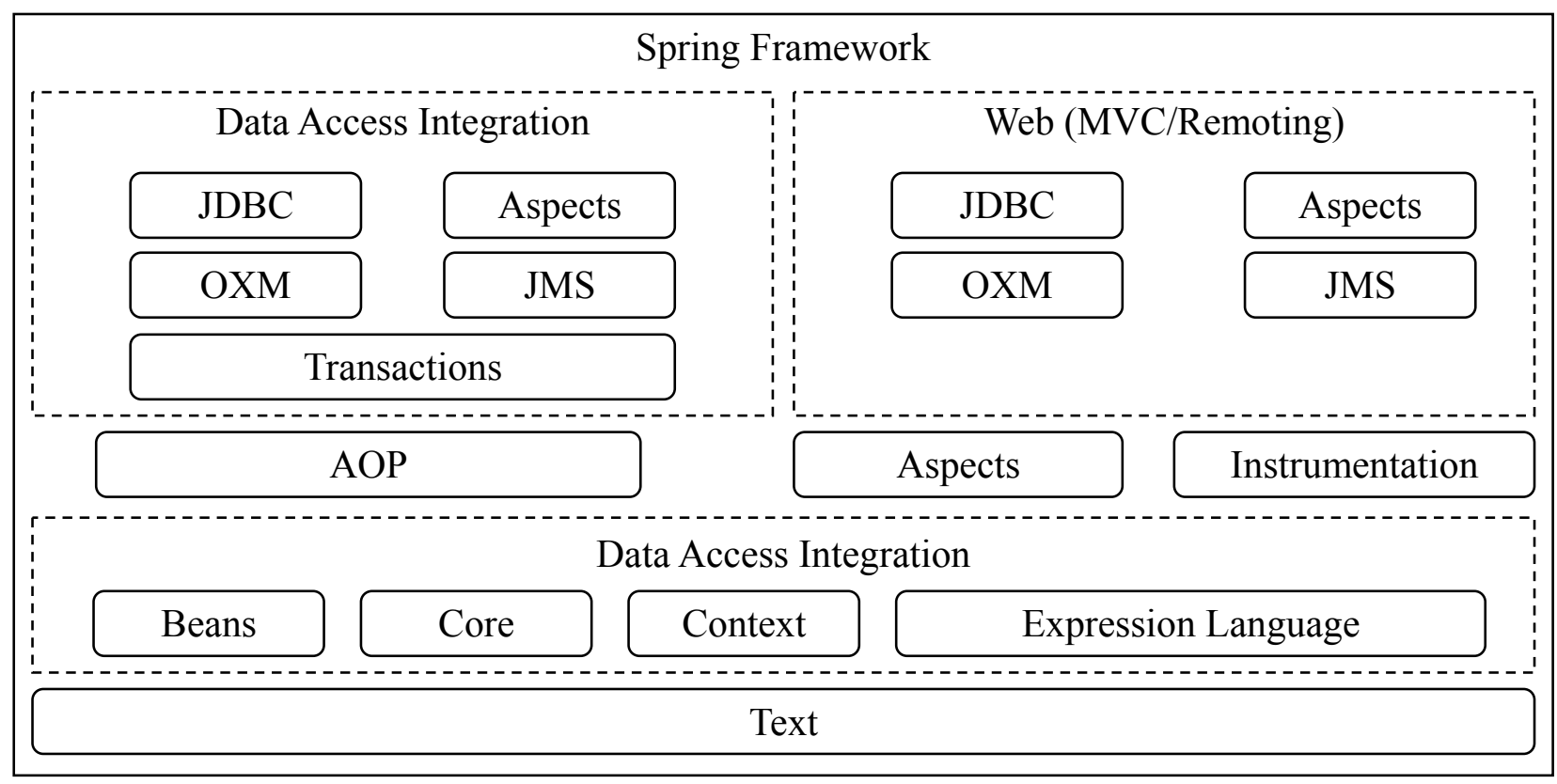

Fig. 1. Architecture on Spring framework

Spring data access includes JDBC, ORM, OXM, JMS and Transactions module. Wherein, JDBC module, which provides no longer need to be a lengthy JDBC encoding related JDBC abstraction layer; ORM module, is a popular object-relational mapping API, including JPA, JDO, Hibernate and iBatis; OXM module, provides a support object /XML mapping to achieve JAXB and Castor, use XMLBeans, JiBX and XStream abstraction layer; JMS module, contains functions for the production and consumption of information; Transactions module, supports programming and declarative transaction management to achieve a special interface class.

Spring Framework has the following advantages: Spring use POJO to develop enterprise applications, using only the advantages of POJO, and not need EJB container products; Spring is organized in a modular way, although a lot of packages and classes, but often used rarely; Spring takes advantage of existing Java technologies, including ORM frameworks, logging frameworks, JEE, Quartz and JDK timers, as well as other view technologies; written test Spring application is very simple, because the environment related code is moved to the frame. By using JavaBean style of POJO, easier to use dependency injection test data; Spring Web framework is a well-designed MVC framework that can replace Struts or other over-designed or unfashionable Web framework; Spring provides a convenient API translation technology; Spring provides a consistent transactional management interface that can be extended to local or global transactions.

\section{JDBC Access to the Database Process}

JDBC is a Java API for executing SQL statements. It can provide unified access to multiple relational databases, which is composed of a set of classes and interfaces written in Java language. JDBC access to database process is shown in Fig. 2.

JDBC provides a benchmark to build more advanced tools and interfaces, so that database developers can write database applications. JDBC for Java programmers are API, for the service provide that achieve the database connection is the interface model. As API, JDBC provides a standard interface for application development, and provides a standard method for the database vendors and third-party middleware vendors to realize the connection to the database. Java has a sturdy, safe, easy to use, and easy to understand and automatically downloaded from the Internet and other characteristics, is an outstanding language to write database applications. It required only 
the method that is dialogue between Java applications with a variety of different databases. And JDBC is a mechanism for this purpose.

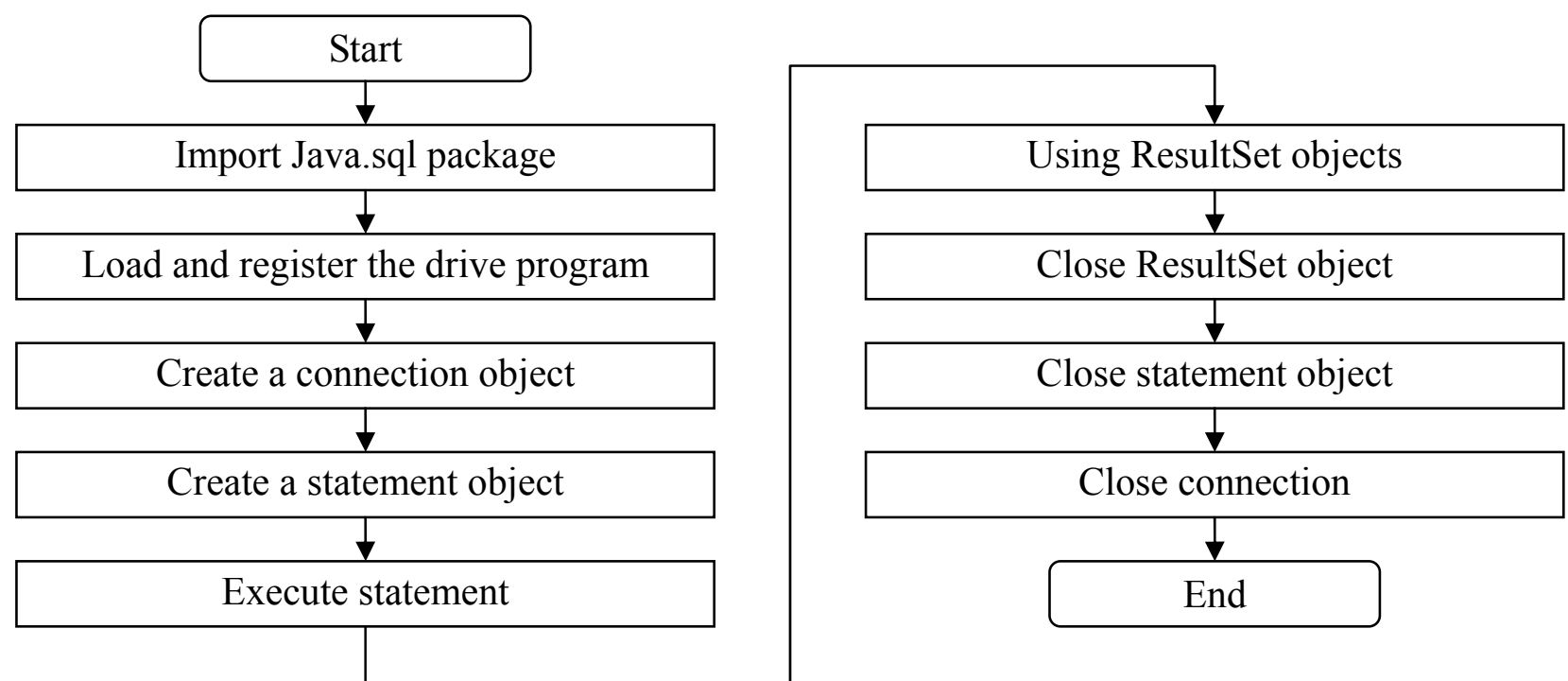

Fig. 2. JDBC Access to the Database Process

\section{Database Design}

Tourism resources management system is a kind of practical software to solve the internal resources of the tourism company. It has a strong practical, that can be used as a separate system, it can also be used as an important part of the tourism enterprise integrated business management system, and the basic thinking of its management is based on geographical information, tourist attractions resource management, tourism hotel resources, tourism resources restaurants, tourism and entertainment resources, tourism routs resources, etc [3]. Database design typically includes conceptual structure design, logical structure design and physical structure design. This article only carries on logical structure design. The main task of the database logical structure design is to transform the data model of the concept layer into the data model of the organization layer, logical structure that is can handle according to the concept structure export specific database management system. The logical structure of database management system design based on DB2 is shown in Table 1 (due to tourism resource management system involves more data tables, only "user" and the "tourist attractions table" as an example).

Table 1. Database logical structure design

\begin{tabular}{clcc|clcc}
\hline \multicolumn{4}{c|}{ User table } & \multicolumn{4}{c}{ Tourist attractions table } \\
\hline No & Field name & Data type & Byte & No & Field name & Data type & Byte \\
\hline 1 & UserID & Varchar & 20 & 1 & AttractionsNumber & Varchar & 20 \\
\hline 2 & UserName & Varchar & 20 & 2 & AttractionsName & Varchar & 50 \\
\hline 3 & UserGender & Char & 2 & 3 & AttractionsAddress & Varchar & 100 \\
\hline 4 & BirthDate & Date & 10 & 4 & ContactPerson & Varchar & 20 \\
\hline 5 & GuideCardNumber & Varchar & 30 & 5 & ContactPhone & Varchar & 50 \\
\hline 6 & UserPhone & Varchar & 50 & 6 & ContactEmail & Varchar & 100 \\
\hline 7 & UserAddress & Varchar & 100 & 7 & ContactAddress & Varchar & 100 \\
\hline 8 & WorkDatme & Date & 10 & 8 & Postcode & Char & 6 \\
\hline 9 & GraduationSchool & Varchar & 50 & 9 & AdultPrice & Decimal & 6,2 \\
\hline 10 & LearningSpecialty & Varchar & 50 & 10 & ChildPrice & Decimal & 6,2 \\
\hline 11 & UserProfile & Clob & 8 & 11 & AttractionsProfile & Clob & 8 \\
\hline
\end{tabular}




\section{Implementation on Data Access of JDBC}

Spring JDBC provides a number of methods and the corresponding different classes to interact with the database. The most classic and popular is the use of JdbcTemplateClass framework. JdbcTemplate class execute SQL queries, update statements and stored procedure calls, In the result set and the extracted return parameters values are iterated. Also catch JDBC exceptions and convert them to generic, information is more abundant, defined hierarchy in org.springframework.dao package. Examples of JdbcTemplate class are a thread that is configured. You can configure an instance of a JdbcTemplate, and then safely inject this shared reference into multiple DAO. When using JdbcTemplate class, the usual practice is to configure a DataSource in the Spring configuration file, then dependency injection the shared data source to the DAO class, JdbcTemplate or in the setter data source is created.

(1) Configure JDBC transaction manager. Transaction management plays a vital role in applications, which is a working unit consisting of a series of tasks, in this working unit, all tasks must be performed simultaneously [4,5]. Only two kinds of possible execution result, either fully implemented all tasks succeed or fail to perform all tasks of all. Spring's transaction manager implements PlatformTransactionManager interface, configure JDBC transaction manager code as follows:

$<$ bean id="touristResources"

class="org.springframework.orm.hibernate3.HibernateTransactionManager">

$<$ property name $=$ "sessionFactory" $>$

$<$ ref bean="sessionFactory" $>$

$<$ property $>$

$</$ bean $>$

(2) Allow Spring to inject DataSource for DAO, the configuration file as follows:

$<$ bean id="dataSource" class="org. apache.commons.dbcp.BasicDataSource" $>$

$<$ property name $=$ "driverClassName" value $=$ "com.mysql.jdbc.Driver" $><$ property $>$

$<$ property name="url" value="jdbc:mysql://localhost:3306/studb? characterEncoding=utf8">

$<$ property $>$

$<$ property name $=$ "username" value $=$ "tongjifu" $><$ property $>$

$<$ property name $=$ "password" value $=" 61616161 "><$ property $>$

$<$ property name $="$ initialSize" value $=" 50 "><$ property $>$

$<$ property name $="$ maxActive" value $=" 80 "><$ property $>$

$</$ bean $>$

$<$ bean id="touristAattractionsDAO" class="com.AthleteIndex.dao.touristAattractionsDAO">

$<$ property name $=$ "dataSource" ref $=$ "dataSource" $><$ property $>$

$<$ bean $>$

(3) The entity class constructs [6]. Entity class is commonly known as POJO, mainly as a present category on the data management and business logic processing level, this class does not implement an interface under the special framework, only as a data container used to store persistent data. Entity class code is as follows:

public class TouristAattractions \{

private String attractionsNumber; private String attractionsName; private String attractionsAddress; private String contactPerson; private String contactPhone; private String contactEmail; private String contactAddress; private String postcode;

private BigDecimal adultPrice;

private BigDecimal childPrice;

private String attractionsProfile;

public String get AttractionsNumber() \{

return this.attractionsNumber; $\}$

public void set AttractionsNumber (String attractionsNumber)

\{this.attractionsNumber $=$ attractionsNumber;

//Omit the getter/setter method of the other attributes \}

(4) Write the DAO implementation. The DAO of Spring JDBC inherits from JdbcDaoSupport class provided by Spring. The DAO implementation based on JDBC, simplify the operation of the 
JDBC, the acquisition and release of JDBC object, the transformation of exception type. DAO implementation code as follows:

package cn.wode.springcore.jdbc; import java.sq1.ResultSet

import java.sql.SQLException;

import java.util.List;

import javax.sql.DataSource;

ITouristAattractionsDAO

\{ public List getTouristAattractions () \{

String sql="SELECT

"attractionsNumber, attractionsName, attractionsAddress, contactPerson, contactPhone, contactEmail, contactAddress, postcode, adultPrice, childPrice, attractionsProfile"; return super.getJdbcTemplate().query

(sql, new

TouristAattractionsRowMapper()); protected class TouristAattractionsRowMapper implements RowMapper \{ public Object mapRow(ResultSet rs, int rowNum) throws SQLException \{

Item item = new Item();

item.setAttractionsNumberNo(rs.getString("attractionsNumber")); item.setAttractionsName (rs.getString("attractionsName")); item.setAttractionsAddress (rs.getString("attractionsAddress")); item.setContactPerson (rs.getTimestamp("contactPersonB")); item.setContactPhone(rs.getString("contactPhone")); item.setContactEmail (rs.getTimestamp("contactEmail")); item.setContactAddress (rs.getTimestamp("contactAddress")); item.setPostcode (rs.getString("postcode")); item.setAdultPrice (rs.getBigDecimal("adultPrice")); item.setChildPrice (rs.getBigDecimal("childPrice")); item.setAttractionsProfile (rs.getBigDecimal("AttractionsProfile")); return item; 


\section{Conclusion}

Tourism resources management system is a computer system, oriented tourists, tour operators and travel managers and travel planners, based on tourism resource information database, in computer hardware and software support, using the theory and methods of systems engineering and information science, which integrates information acquisition, storage, processing, analysis, display and fast transmission by using computer technology, network technology, and geographic information system technology [7]. JDBC uses the existing SQL standards and supports connectivity standards of other databases, such as the bridge between ODBC. JDBC implements all of these goals of oriented standards and has a simple, strict type definition, and high-performance implementation interface. The research content of this paper is to solve key technical problems of systems development, which is of great significance to improve the efficiency of system development.

\section{References}

[1] L. Y. Yang, "Design and implementation on Qingyuan mountain scenic tourist resources management information system Based on GIS," Master's degree of Huaqiao University, 2011.

[2] J. Du, L. J. He, H. J. Ouyang "Design and Implementation of Online Examination System Based on JDBC and DAO Mode," Journal of Xianyang Normal University, vol. 29, no. 2, pp. 26-29, 2014.

[3] J. F. Tong, "Data persistence on tourism resources management system based on NHibernate," Electronic Test, vol. 21, no. 20, pp. 12-13, 2014.

[4] W. Y Zhang, M. J. Xu, Y. Xue, "Introduction to Spring Zero Configurationand XML Configurationand," Computer Systems \& Applications, vol. 24, no. 2, pp. 270-275, 2015.

[5] J. H. Xu, B. Song, R. Ding, "Research of Spring MVC+Hibernate+jQeury Development Framework Application," Techniques of Automation and Applications, vol. 34, no. 3, pp. 42-46, 2015.

[6] L. Zhang, W. X. Zhang, "Application of Vegetables Transportation and Sales Integrated Management System in Date Persistent Based on Spring and JDBC," Journal of Anhui Agricultural Sciences, vol. 40, no. 7, pp. 4401-4403, 2012.

[7] J. F. Tong, "Requirement and design on tourism resources management system," Electronic Test, vol. 21, no. 16, pp. 68-69, 2014. 